\title{
Deterministic mathematical morphology for CAD/CAM
}

\begin{abstract}
Purpose

This paper presents a new geometric model based on the mathematical morphology paradigm, specialized to provide determinism to the classic morphological operations. The determinism is needed to model dynamic processes that require an order of application, as is the case for designing and manufacturing objects in CAD/CAM environments.

Design/methodology/approach

The basic trajectory-based operation is the basis of the proposed morphological specialization. This operation allows the definition of morphological operators that obtain sequentially ordered sets of points from the boundary of the target objects, inexistent determinism in the classical morphological paradigm. From this basic operation, the complete set of morphological operators is redefined, incorporating the concept of boundary and determinism: trajectory-based erosion and dilation, and other morphological filtering operations.

Findings

This new morphological framework allows the definition of complex threedimensional objects, providing arithmetical support to generating machining trajectories, one of the most complex problems currently occurring in CAD/CAM.

Originality/value

The model proposes the integration of the processes of design and manufacture, so that it avoids the problems of accuracy and integrity that present other classic geometric models that divide these processes in two phases. Furthermore, the morphological operative is based on points sets, so the geometric data structures and the operations are intrinsically simple and efficient. Another important value that no excessive computational resources are needed, because only the points in the boundary are processed.
\end{abstract}

Keywords: Mathematical morphology; Geometric model; Boundary-based morphology; Deterministic morphology 


\section{Introduction}

Mathematical morphology was born in 1964 when scientists Georges Matheron and Jean Serra applied the fundamental ideas of Minkowsky and Hadwiger to their studies on quantification of mineral characteristics (Serra, 1982). In 1988, Jean Serra made a generalization of the mathematical morphology in a theoretical framework based on complete lattices (full set of points arranged with upper bound [supremum] and lower bound [infimum]). This generalization brought flexibility to the theory, which meant that it could be applied to a greater number of structures and fields of application (Serra, 1988)

Mathematical morphology is based on set theory. The sets represent shapes of objects in an $n$-dimensional space. A series of standardized morphological operations are applied to these sets. These operations are based on geometric relationships between the sets' points. The aim of the morphological operations is to transform a set of points that gives shape to an object from another set of points called the structuring element.

References about mathematical morphology are in their abundance in various productive sectors. A good review of these applications can be found in (ISSM, 2001) where the following appear: navigation systems, industrial control, medicine and biology, physics, aeronautics, real-time systems or restoration processes. Image processing is one of the main uses of mathematical morphology. In (Ghosh and Deguchi, 2008), (Plaza et al., 2009) and (Salember et al., 2009) are shown recent techniques that apply mathematical morphology to image processing.

In an industrial environment, the process of manufacturing a piece is based on the manipulation of a primitive object via contact with a tool that transforms the object progressively, obtaining the desired design. The analogy with the morphological operation of erosion is obvious. Nevertheless, few references about the relation between the morphological operations and the process of design and manufacture, few references can be found. We presume that the non-deterministic nature of classic mathematical morphology makes it very difficult to adapt their basic operations to the dynamics of concepts such as the ordered trajectory. The morphological operation is not based on temporary orders since their original ones act on continuous sets of points and produce new continuous sets of points as a result, without establishing a path order on its elements. This order relationship is necessary when the morphological paradigm must stage dynamic processes such as the trajectory process. In addition, the morphological operation always obtains complete results, without being able to apply partial transformations on objects that are involved in the operation.

A formal framework is presented below, which provides a specialization of the classic morphological operations, giving them the determinism of dynamic processes that require an order of application, as is the case for designing and manufacturing articles in CAD/CAM environments. This model is inspired by the classic morphological paradigm that formally defines objects from their boundary and applies morphological operations that transform these objects.

Firstly, the general morphological framework is proposed, and the objects, structuring elements and the set of morphological operations that are to participate in the paradigm are defined. Then, the basic trajectory-based operation is detailed. This 
is an operation that forms the basis of specialization that gives determinism to the morphological operation and the basic morphological operators of trajectory-based erosion and dilation are defined. They serve as the basis for defining morphological filters. A discussion section is then introduced, to analyze other attempts to use the morphological concepts in $\mathrm{CAD} / \mathrm{CAM}$ and to validate the proposed model in this context. The final section presents the findings and conclusions from the research and the used references.

\section{Deterministic mathematical morphology (DMM)}

The proposed formal morphological framework $D M M$ is defined by the following expression:

$$
D M M=\langle E, O B, E E, O P\rangle
$$

$E \quad$ Represents the space of representation of the sets involved in the model.

$O B$ Represents the set of objects to be transformed by morphological operations.

EE Represents the set of structuring elements through which the morphological operations are to be carried out.

$O P$ Refers to the set of morphological operations for transforming the set of objects $O B$ using $E E$ objects.

$O B$ and $E E$ sets are complete lattices of the Euclidean space $E$, which define sets of geometric points of the workspace. In the case of two-dimensional objects $E \equiv R^{2}$, and three-dimensional objects $E \equiv R^{3}$. In general, $E \equiv R^{n}$. The proposed morphological operation is not restricted to a two-dimensional or three-dimensional space but is applicable to any space $R^{n}$. To facilitate the representation, this article uses two- and three-dimensional figures, always being particular cases of the general set.

The set of structuring elements $E E$ is determined by those objects that are centered on the origin of Euclidean space coordinates, i.e.:

$$
E E=\left\{B_{x}: x=0,0 \in R^{n}\right\}
$$

Expression 2.2

The set of objects to be transformed $O B$ shall consist of those objects $X$ whose center $c$ has moved with regard to the origin of coordinates:

$$
O B=\left\{X_{c}: c=0+d, d \in R^{n}\right\}
$$

Expression 2.3

Compared to the solid modeling presented by mathematical morphology, the proposed model is a surface model, i.e., it only works with objects' surface information, without any information concerning its interior. Given these considerations, the objects' relevant geometric information is located on its contour or boundary. The model uses this to characterize the objects through its contour, but in such a way that this characterization does not result in a loss of generality. 
Function $\operatorname{In}(A)$ is defined to retrieve the set of points located inside a set $A$. The function obtains the set of positions in which the center of an $n$-solid ball can be placed so that it is positioned inside the object $A$ :

$$
\begin{gathered}
\operatorname{In}(A)=\{x \in E / \exists \varepsilon>0: B(x, \varepsilon) \subset A\} \\
B(x, \varepsilon)=\{n-\text { ball with center } x \text { and radius } \varepsilon\} \\
\text { Expression } 2.4
\end{gathered}
$$

The function that associates a set with its boundary or contour is called $\operatorname{Fr}(A)$ and shall consist of the set of points belonging to the contour of the object $A$. This function is only the result of applying the boundary extraction morphological filter $\left(\beta_{0}\right)$ (González and Woods, 2008), which is given by the remainder of object $A$ with the set of interior points of the object:

$$
\operatorname{Fr}(A)=\underset{\text { Expression 2.5 }}{\beta_{O}=A-\operatorname{In}(A)}
$$

Differential morphology is given by the following expression:

$$
\begin{gathered}
A-B=\{x \mid x \in A, x \notin B\}=A \cap B^{c} \\
B^{c}=\left\{\begin{array}{c}
\text { complementary of } B\} \\
\text { Expression } 2.6
\end{array}\right.
\end{gathered}
$$

Once the objects to be transformed and the structuring elements that will carry out the transformations have been formally defined, the set of operations that will finally transform the set of objects $O B$ from the set of objects $E E$ must be formally defined.

In conventional mathematical morphology, the operations that transform objects are defined as a sequence of operations that act on the objects (Serra, 1982). No application order is set for these basic, elementary operations.

So that the proposed model defines morphologically deterministic processes, the morphological paradigm must incorporate a specialization of the morphological operations that adapt them to this type of process. The determinism provided to the operation will ensure that the model comply with its functional purpose.

The set of morphological operations that transform the set of objects $O B$ from the set of objects $E E$ is defined by the following expression:

$$
\begin{gathered}
\mathrm{OP}=\left\langle\mathrm{OP}_{\mathrm{ND}}, \mathrm{OP}_{\mathrm{D}}\right\rangle \\
\text { Expression } 2.7
\end{gathered}
$$

$\mathrm{OP}_{\mathrm{ND}}$ Represents the set of non-deterministic operations of classical nonlinear mathematical morphology.

$\mathrm{OP}_{\mathrm{D}}$ Represents the set of specialized morphological operations, equipped with an order of application.

Below, the basic trajectory-based operation is defined on which the deterministic specialization of the basic morphological operations and their associated filters will be based. 


\subsection{Basic trajectory-based operation}

The basic trajectory-based operation $\diamond_{\tau}$ constitutes the basis of the proposed morphological specialization. This morphological operation allows the definition of morphological operators that obtain sequentially ordered sets of points, inexistent determinism from the classical morphological paradigm point of view.

To guide the orderly generation of results, the operation obtains the set of points by repeatedly applying another fundamental basic operation, called Instantaneous Basic Operation $\left(\vartheta_{\tau(\mathrm{k})}\right)$. This operation is called instantaneous because it only calculates a point of the total set of points that would be obtained after implementing a conventional morphological operation.

The sequential application of instantaneous morphological operations will form the basic trajectory-based operation that will orderly obtain the classic morphological operation's complete set.

\subsubsection{Trajectory function}

A real parameter function $\varsigma$ is defined, which obtains the full set of orientationspositions that must be applied to the object to be transformed, to help the structuring element come into contact with the complete set of accessible points that form the boundary of the object, as it moves in the direction of the ordinate axis.

For a $k$ value of the normalized space [0.1], the function $\varsigma$ returns a pair of values (orientation, position), which define the transformations that must be performed on the object to ensure that a particular boundary point of the object is accessible to the structuring element.

The complete scan of the parametric space will describe the complete sequence of positions and orientations that must be applied to the object so that the structuring element can come in contact with the total set of points that form the object's boundary. This series of positions and orientations is ordered by a neighborhood criterion in the points, so that the normalized space's sequential scan will detail the sequence of transformations to be made so that the structuring element can touch the complete set of object's boundary points in an orderly manner (Figure 1). 

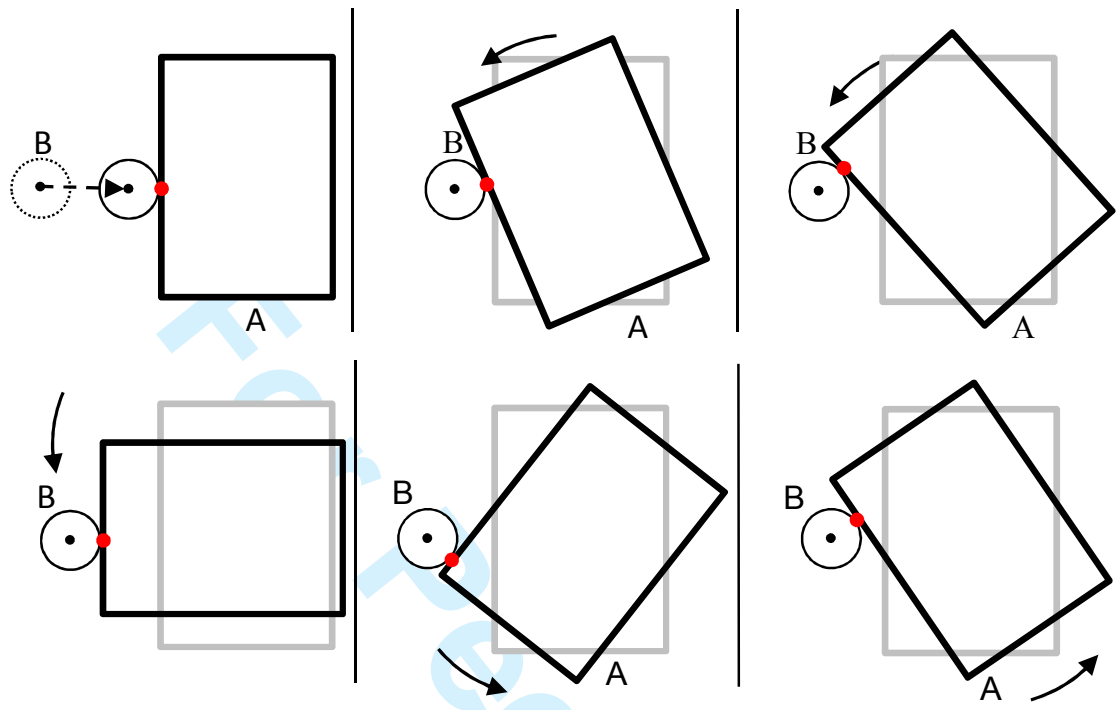

Figure 1. Different points of contact of $B$ with $A$ after changing A's orientation

At this point, it is important to emphasize that deciding that the structuring element moves in one direction on the object to be transformed does not correspond to a restriction of the system, but to a consideration that helps ordered generation of results, a specialization that allows determinism to be added to the morphological paradigm.

Once the transformations needed so that the morphological operation can obtain complete and ordered results are known, the functions that apply these transformations to the entire set of points that form the object's accessible boundary to be transformed must be defined.

The trajectory function is defined in a point $\mathrm{T}(p, k)$ that applies the transformations of rotation and translation to ensure that a particular point $p$ of the object will be accessible by the structuring element. The formal definition of the trajectory function of a point $T$ is as follows:

$$
\begin{aligned}
& \mathrm{T}: \mathrm{R}^{\mathrm{n}} \mathrm{x} \mathrm{R} \rightarrow \mathrm{R}^{\mathrm{n}} \\
& \mathrm{T}(\mathrm{p}, \mathrm{k})=\mathrm{p}^{\prime} \rightarrow \operatorname{Pos}(\varsigma(\mathrm{k})) \cdot(\operatorname{Rot}(\varsigma(\mathrm{k})) \cdot \mathrm{p})
\end{aligned}
$$

Rot is a rotation matrix generated from the orientation vector, which provides the function $\varsigma$ for the parametric value $k$ and $P o s$ is a translation matrix generated via the position returned by the function $\varsigma$ for the same parametric value.

Having specified the trajectory function for a point, defining a new function that extends the trajectory definition in a point to the full set of points that form an object 
is trivial. The trajectory function in an object $\tau(\mathrm{A}, \mathrm{k})$ will be given by applying the trajectory function for the entire set of points that constitute the object:

$$
\tau(A, k)=\underset{\text { Expression } 2.9}{\tau: P\left(R^{n}\right) \times R \rightarrow P\left(R^{n}\right)}=\underset{T(q, k)\}}{\{p \in E / \forall q \in A p}
$$

The trajectory function in an object orients and positions the object so that a particular point of it is accessible by the structuring element, as it moves in the direction of the axis of ordinates. Due to the fact that the transformations applied only make rotations and translations, the object will not suffer scaling transformations or deformations. As a result, the operation maintains the object's shape even though its orientation and position are changed with respect to the structuring element in the representation space.

The sequential application of the trajectory function in the normalized parametrical space will transform the object in an orderly manner as the actual parameter starts taking consecutive values. The complete scan of the normalized space will ensure that all of the object's accessible boundary points will come into contact with the structuring element as it moves in the direction indicated by the axis of ordinates (provided that the geometry of the object and the structuring element allow this action).

\subsubsection{Instantaneous basic operation}

The proposed instantaneous basic operation $\left(\vartheta_{\tau(\mathrm{k})}\right)$ is a basic morphological operation that includes a $k$ parameter that indicates its position within the total set of elementary operations that will compose the entire basic trajectory-based operation. The operation is called instantaneous because it obtains a single point of the total set of points that would be obtained following the application of a conventional morphological operation.

In descriptive terms, the instantaneous basic operation obtains the center of the structuring element when it is moved a distance dist following a direction $\vec{v}$ until it touches a boundary object $A$ to which the trajectory function has been applied. This operation represents the approach of the structuring element to the object in a morphological transformation process.

In particular, the instantaneous basic operation is formulated as follows:

$$
\begin{aligned}
\mathrm{A} \diamond_{\tau(\mathrm{k})} \mathrm{B}=\mathrm{p} \in \mathrm{E}, \mathrm{p}= & \tau^{-1}(\mathrm{q}, \mathrm{k}), \mathrm{q}=\operatorname{dist}_{\overrightarrow{\mathrm{v}}}(\mathrm{B}, \tau(\mathrm{A}, \mathrm{k})) \cdot \overrightarrow{\mathrm{v}} \\
\tau^{-1} & =\{\text { inverse of } \tau\} \\
& \text { Expression 2.10 }
\end{aligned}
$$

Each of the instantaneous operations performs homogeneous transformations in the object, rigid body transformations defined in the trajectory function, which transformed the objects without scaling or distorting them. The inclusion of the trajectory function in the instantaneous operation ensures that the distance is always calculated between the structuring element and an accessible point of the boundary of the object that is to be transformed. 
The function of distance $\operatorname{dist}_{\vec{v}}(X, Y)$ obtains the distance that at least one object $X$ has to move to come into contact with another object $Y$ following a certain direction $\vec{v}$. This distance is given by the minimum distance between the two objects in the direction that the given vector determines (Jimeno, 2003). In formal terms:

$$
\operatorname{dist}_{\overrightarrow{\mathrm{v}}}(\mathrm{B}, \mathrm{X})=\min \left(\mathrm{d}_{\overrightarrow{\mathrm{v}}}(\mathrm{b}, \mathrm{X})\right), \forall \mathrm{b} \in \mathrm{B}
$$

Expression 2.11

The function $\mathrm{d}_{\overrightarrow{\mathrm{v}}}$ obtains the distance between a point and an object in the direction that indicates the vector $\vec{v}$. Geometrically the function $\mathrm{d}_{\vec{v}}(b, X)$ obtains the Euclidean distance between the point $b$ and the closest point of the object, obtained as the intersection of the line that defines the vector $\vec{v}$ and that passes through this point and the object $X$. Figure 2 shows an example of application of the dist $\overrightarrow{\vec{d}}$ function.

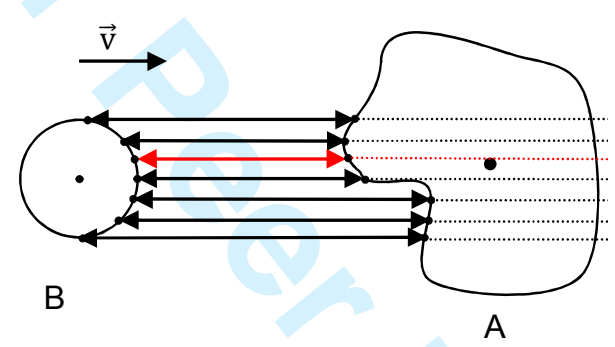

Figure 2. Calculation of the distance function between two objects $A$ and $B$ in the direction of vector $\overrightarrow{\mathbf{v}}$.

The trajectory function of an object modifies the position and orientation of the set of boundary points of an object through the application of rotation and translation matrices. Applying the inverses of these matrices is enough to undo these changes.

The inverse trajectory function in a point $\mathrm{T}^{-1}$ applies the inverse transformation matrices of rotation and position defined in the trajectory function to a point.

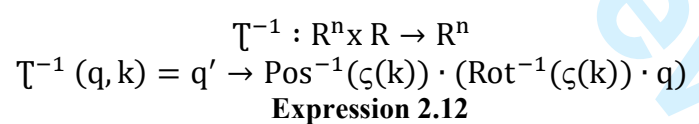

Figure 3 shows the application of the instantaneous basic operation on an object $A$ as a series of four phases: a first phase (a) in which the structuring element $B$ appears to be a distance from the object to be transformed $A$, a second phase (b) in which the trajectory function is applied to the object $A$ for a determined $k$ value, a third phase (c) in which the distance that the center of the structuring element has to move to come into contact with the object is calculated, and a final stage (d) in which the inverse trajectory function is applied to the center of the tool used to calculate the end $p$ point that the morphological operation obtains. 
aa

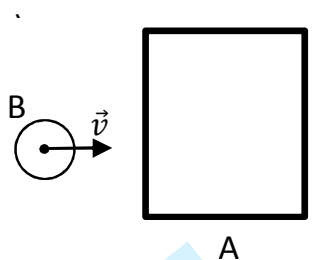

c)

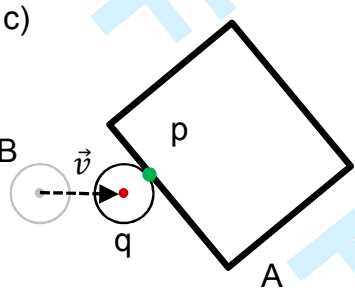

b)

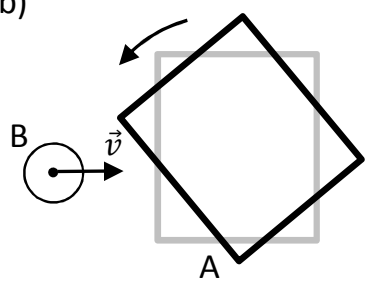

d)

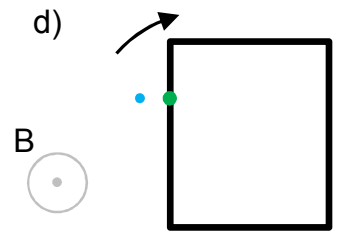

A

Figure 3. Geometric description of the instantaneous basic operation. A is the piece,

$B$ is the structuring element, $p$ is a point in the surface, $q$ is the center of the structuring element and $\vec{v}$ is the direction of application

\subsubsection{Basic trajectory-based operation}

Having defined the instantaneous operation $\diamond_{\tau(\mathrm{k})}$ which obtains a specific point in the parametrical space in which the object's complete transformation is defined, the repeated application of these instantaneous basic operations throughout the complete parametric space will define the basic operation with complete trajectory $\left(A \diamond_{\tau} B\right)$ by itself.

$$
\mathrm{A} \diamond_{\tau} \mathrm{B}=\bigcup_{\mathrm{k} \in[0 \ldots 1]} \mathrm{A} \diamond_{\tau(\mathrm{k})} \mathrm{B}
$$

Expression 2.13

Sequentially applying instantaneous operators in all the parametric $k$ range ensures that the morphological operator is fully implemented. The parametric $k$ value is normalized meaning that all of the transformations are ordered according to the parameter with the initial position $k=0$ and the final position $k=1$. In the generation of results, this order is associated with the ordered set that establishes the trajectory function. The basic trajectory-based operation links a sequence of morphological operations, establishing a determinism that will provide an orderly movement in the space, a determinism that does not exist in the classic morphological paradigm.

Furthermore, it is important to emphasize once more that the objects involved in the morphological translations and rotations applied in the basic trajectory-based operation do not vary in size and shape; only their position and orientation in space alter. The purpose of the instantaneous basic operation is not to change the shape of objects, only sequence results generated by the morphological transformation 
operations, which will be responsible for modifying the shape of the objects $O B$, reproducing the deterministic systems' processes.

\subsubsection{Basic partial trajectory-based operations}

The trajectory function $\tau$ ensures that the basic operator is completely applied to the entire set of points that form the accessible boundary of the object ordered according to the parametric space $\mathrm{k}=0 \ldots 1$. However, parametric space trajectories can be defined that do not fill the entire parametric space forming subsets of the complete morphological operation. The partial path of parametric space constitutes the partial trajectory-based operation $\left(A \diamond_{\tau<i, j>} B\right)$ :

$$
A \diamond_{\tau<i, j>} B=\bigcup_{\substack{k \in[i \ldots . j] \\ \text { Expression } 2.14}} A \diamond_{\tau(k)} B, 0 \leq i<j \leq 1
$$

\subsection{Deterministic morphological operations}

Having defined the basic, partial or complete trajectory-based operation, the next step is to integrate this operation in the classic morphological operation, thus incorporating a set of morphological operations $\left(O P_{D}\right)$ to the model, which are equipped with the determinism and which transform an object from a structuring element.

There are two fundamental morphological operations in classic mathematical morphology: erosion and dilation. Both operations are the basis for the definition of morphological filtering operators. They are formally defined below, and the specialization that the model performs to equip them with a determinism is specified.

\subsubsection{Trajectory-based erosion}

In mathematical morphology, the erosion operation can be defined by the equation:

$$
\begin{gathered}
\mathrm{A} \ominus \mathrm{B}=\left\{\mathrm{y} \in \mathrm{E}, \mathrm{B}_{\mathrm{y}} \subseteq \mathrm{A}\right\} \\
\text { Expression } 2.15
\end{gathered}
$$

A descriptive interpretation of the operation defines it as the place of the positions of structuring element $B$ 's center when it is included in $A$. The erosion contour is defined by the structuring element's centers when it touches the inner edge of the object.

The morphological erosion acts on the set of points of $A$ consequently producing a new set of points, transformed without establishing a trajectory order on its elements. This ordered set is needed when the morphological paradigm has to stage deterministic dynamic processes.

The model incorporates a specialization of the morphological erosion operation based on the use of the instantaneous basic operation in order to provide the morphological erosion with that necessary determinism.

By integrating the instantaneous basic operation in the erosion operation, the final complete erosion set is obtained by repeatedly applying variable real parameter 
instantaneous basic operations, defining instant erosion $\ominus_{\tau(\mathrm{k})}$ as a step in the morphological erosion:

$$
A \ominus_{\tau(k)} B=p \in E: p=\tau^{-1}(q, k), q=\operatorname{dist}_{\vec{v}}(B, \tau(A, k)) \cdot \vec{v} \wedge B_{q} \subseteq \tau(A, k)
$$

Expression 2.16

At this point, an explanation about the distance function dist $\overrightarrow{\vec{v}}$ is needed, which calculates the minimum distance between the object and the structuring element. For morphological trajectory-based erosion, the morphological erosion definition requires the structuring element $B$ to be completely included in the object $A$. The distance function integrated into the morphological erosion must calculate the minimum distance from the object $A$ to the element $B$ respecting the restriction of placing the structuring element within the object. This situation does not occur for morphological operators such as dilation in which the structuring element must touch the object from the outside of it.

Trajectory-based erosion $\ominus_{\tau}$ is defined as the set of points obtained by repeatedly applying the instantaneous erosion $\ominus_{\tau(\mathrm{k})}$ for the real domain $[0 \ldots 1]$.

$$
A \ominus_{\tau} B=\bigcup_{k \in[0 . \ldots 1]}\left(A \ominus_{\tau(k)} B\right)=\left\{p \in E: p=\tau^{-1}(q, k), q=\operatorname{dist}_{\vec{v}}(B, \tau(A, k)) \cdot \vec{v} \wedge B_{q} \subseteq \tau(A, k)\right\}
$$

Expression 2.17

The trajectory function $\tau$ ensures a path inside object $A$ in the normalized space [0...1]. If the real variable $k$ runs throughout the interval, the object's complete erosion boundary is obtained as a result, since it will have obtained all of the structuring element's centers when it touches the object.

At this point, the difference between classic morphological erosion and trajectorybased erosion can be observed. While the result for classic morphological erosion was a set of continuous points representing the eroded object's contour and its inside, the trajectory-based erosion only works the contours of the eroded object, in an orderly manner, with surface information of the objects obtained, without there being any information on its interior (Figure 4).

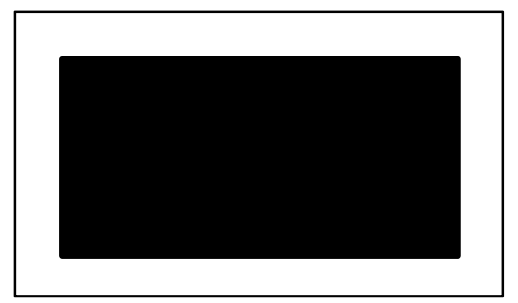

Classic morphological erosion

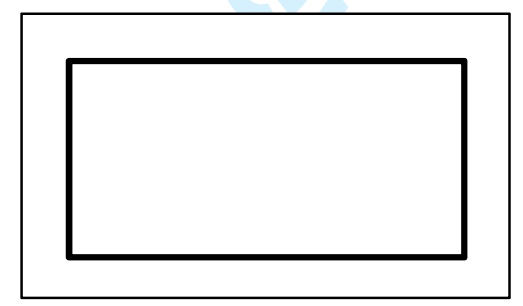

Trajectory-based morphological erosion

Figure 4. Classic morphological erosion vs. Morphological trajectory-based erosion 
Even though both operations do not return the same set of points, the morphological erosion boundary and trajectory-based erosion do coincide: If $A$ and $B$ are two sets included in $E$, then the trajectory-based erosion is equal to the erosion's boundary:

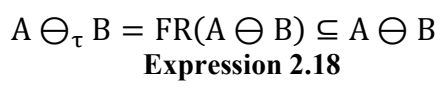

This fact can be proven by the formal proof method of reduction ad absurdum.

Proof $A \ominus_{\tau} B \subseteq F R(A \ominus B)$

Supposing that the following hypothesis is true: a point of the trajectory-based erosion $A \ominus_{\tau} B$ is not part of the set of points boundary to the object FR(A $\left.\ominus B\right)$. The points obtained by the trajectory-based erosion $A \ominus_{\tau} B$ correspond to the geometric locations of the structuring element's center when it is contained in $A$ and it touches at least one point of its contour.

$$
p=\tau^{-1}(q, k), q=\operatorname{dist}_{\vec{v}}(B, \tau(A, k)) \cdot \vec{v} \wedge B_{P} \subseteq \tau(A, k)
$$

Expression 2.19

Any point that is the result of applying the trajectory-based erosion will therefore be a minimum distance away from the contour of $A$, which allows the structuring element to be positioned on the inside of the object.

Morphological erosion obtains the set of points that position the structuring element within the object without having to evaluate whether the structuring element comes into contact with the object's contour or not. Morphological erosion only ensures that the structuring element is contained in the object, thus morphological erosion will shape all those positions that place the structuring element inside the object touching it's contour or not.

$$
\mathrm{A} \ominus \mathrm{B}=\left\{\mathrm{x} \in \mathrm{R}^{\mathrm{n}}, \mathrm{B}_{\mathrm{x}} \subseteq \mathrm{A}\right\}
$$

The morphological erosion's boundary removes the points that position the structuring element within the object without touching its contour, i.e. the points that are not away from the contour by the minimum distance that allows you to place the structuring element on the inside of the object.

$$
\begin{gathered}
\mathrm{FR}(\mathrm{A} \ominus \mathrm{B})=\mathrm{A}-\mathrm{In}(\mathrm{A}) \\
\operatorname{In}(\mathrm{A})=\mathrm{x} \in \mathrm{E} / \exists \varepsilon>0: \mathrm{B}(\mathrm{x}, \varepsilon) \subset \mathrm{A} \\
\mathrm{B}(\mathrm{x}, \varepsilon)=\{\mathrm{n}-\text { ball with center } \mathrm{x} \text { and radius } \varepsilon\} \\
\text { Expression } \mathbf{2 . 2 1}
\end{gathered}
$$

For a point to belong to the boundary of the morphological erosion $\mathrm{A} \ominus \mathrm{B}$ it must be the minimum distance from the object's contour that allows the structuring element to be positioned inside object A. If a point belongs to the set of points that is the result of applying trajectory-based erosion to the object, the point shall be located at a 
minimum distance from the object $A$ 's contour, such that the structuring element centered on this point shall be contained in $A$.

$$
\begin{aligned}
& \operatorname{dist}(p, A)=\min , \forall p \in A \ominus_{\tau} B \\
& \text { dist }=\{\text { distance between the point } \mathrm{p} y \text { and the object } \mathrm{A}\}
\end{aligned}
$$

If the initial hypothesis that the point does not belong to the morphological erosion's boundary is true, then the point belongs to the inside of the object, which means that the point will be a minimum distance from the object's contour to ensure that the structuring element is contained in the object plus a proportional distance $\delta$ to the radius $\varepsilon$ of the n-ball used in the function $\operatorname{In}(A)$ to calculate the object's inner points:

$$
\begin{aligned}
& \operatorname{dist}(\mathrm{p}, \mathrm{A})=\min +\delta, \delta>0, \forall \mathrm{p} \in \mathrm{FR}(\mathrm{A} \ominus \mathrm{B}) \\
& \text { Expression } 2.23
\end{aligned}
$$

If we make the distances equal in both cases, a clear contradiction is found:

$$
\min =\min +\delta, \delta>0
$$

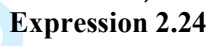

Expression 2.24

The initial hypothesis is therefore false, any point belonging to trajectory-based erosion forms part of the set of points obtained by the classic morphological erosion's contour.

Proof FR $(A \ominus B) \subseteq A \ominus_{\tau} B$

This second implication is proven in an identical manner to the first. We assume that the hypothesis that a point on the classic morphological erosion FR(A $\ominus B$ ) 's boundary does not belong to the set of points that is the result of applying the trajectory-based erosion $\mathrm{A} \ominus_{\tau} \mathrm{B}$. A point belonging to the morphological erosion A $\ominus$ B's boundary must be minimally separated from object $A$ 's contour so that the structuring element is positioned on the inside of the object. However, given that it is not part of the trajectory-based erosion it will not be located a minimum distance from the object's contour, such that the structuring element is positioned on the inside of the object, which is a contradiction. The initial hypothesis is false and as a consequence, the opposite hypothesis is certain:

Given that $A \ominus_{\tau} B \subseteq F R(A \ominus B)$ and $A \ominus_{\tau} B \subseteq F R(A \ominus B)$ are proven, the initial expression is proven to be: $A \ominus_{\tau} B=F R(A \ominus B)$. L.Q.Q.D.

\subsubsection{Partial trajectory-based erosion}

Trajectory-based erosion can control the order that points are obtained in the final set. Having defined the partial ordered set $\leq$ in $E$, an orderly series of parametric $k$ values on the interval $[0 \ldots 1]$ will therefore cause the structuring element's centers to be obtained in an orderly manner, according to the movement defined by the trajectory function $\tau$. 
Although the trajectory-based erosion can obtain the complete erosion's boundary, if the transformation does not cover the entire parametric space $k$ the result of applying the trajectory-based erosion is a partial erosion of the object.

$$
\mathrm{A} \ominus_{\boldsymbol{\tau}<\mathrm{i}, \mathrm{j}>} \mathrm{B}=\bigcup_{\substack{\mathrm{k} \in[\mathrm{i} \ldots . .] \\ \text { Expression } 2.25}} \mathrm{~A} \ominus_{\tau(\mathrm{k})} \mathrm{B}, 0 \leq \mathrm{i}<\mathrm{j} \leq 1
$$

Expression 2.25

\subsubsection{Trajectory-based dilation}

In mathematical morphology dilation is defined by the expression:

$$
\mathrm{A} \oplus \mathrm{B}=\{\mathrm{x} \in \mathrm{E}, \mathrm{B} \mathrm{x} \cap \mathrm{A} \neq \varnothing\}
$$

Expression 2.26

In descriptive terms, this operation can be defined as the place of the structuring element $H$ 's center positions when it touches the set $A$. For example, for $E \equiv R^{2}$ the dilation of a square by a circular object obtains another, bigger square with rounded corners.

We define the instant dilation $\oplus_{\tau(\mathrm{k})}$ as a morphological dilation step:

$$
\mathrm{A} \oplus_{\tau(\mathrm{k})} \mathrm{B}=\mathrm{p} \in \mathrm{E}: \mathrm{p}=\tau^{-1}(\mathrm{q}, \mathrm{k}), \underset{\text { Expression }}{\mathrm{q}}=\operatorname{dist}_{\overrightarrow{\mathrm{v}}}(\mathrm{B}, \tau(\mathrm{A}, \mathrm{k})) \cdot \overrightarrow{\mathrm{v}} \wedge \mathrm{B}_{\mathrm{q}} \cap \tau(\mathrm{A}, \mathrm{k}) \neq \emptyset
$$

For instantaneous dilation, the distance function dist $\overrightarrow{\vec{v}}$ will calculate the minimum distance from object $A$ to object $B$ without establishing the condition that the structuring element is included in the object. The computed distance will always keep the structuring element on the outside of the object.

Trajectory-based dilation is given by the set of points obtained by repeatedly applying the instantaneous dilation $\oplus_{\tau(\mathrm{k})}$ to the real domain $[0 \ldots 1]$.

$$
\begin{gathered}
A \oplus_{\tau} B=\bigcup_{k \in[0 \ldots 1]}\left(A \oplus_{\tau(k)} B\right)= \\
=\left\{p \in E: p=\tau^{-1}(q, k), q=\underset{\text { Expression 2.28 }}{\left.\operatorname{dist}_{\vec{v}}(B, \tau(A, k)) \cdot \vec{v} \wedge B_{q} \cap \tau(A, k) \neq \varnothing\right\}}\right.
\end{gathered}
$$

Trajectory-based dilation's result is an ordered set of points dilated with regard to the original object, which will coincide with the classic morphological dilation's boundary.

As occurred with erosion the classic dilation's boundary can be proven to coincide with the trajectory-based dilation. If $A$ and $B$ are two sets included in $E$, then trajectory-based dilation is equal to the classic morphological dilation's boundary.

$$
A \oplus_{\tau} B=F R(A \oplus B)
$$

Expression 2.29

\subsubsection{Partial trajectory-based dilation}


Again, if the trajectory does not completely cover the parametric space $k$, the result of applying trajectory-based dilation is a partial dilation of the object.

$$
A \oplus_{\tau<i, j>} B=\bigcup_{\substack{k \in[i \ldots . j] \\ \text { Expression 2.30 }}} A \oplus_{\tau(k)} B, 0 \leq i<j \leq 1
$$

\subsubsection{Trajectory-based erosion and trajectory-based dilation, morphological operators of erosion and dilation.}

It is suffice to apply the Adjunction Theorem to formally prove that the erosion and dilation operations presented are effectively such. The Adjunction Theorem details under which conditions a pair of operations is an erosion/dilation pair (Serra, 2001). This theorem is based on the Galois connections that establish particular correspondences between partially ordered sets.

The adjunction theorem states that if two operators $\delta$ and $\varepsilon$ are linked by the equivalence $\mathrm{X} \subseteq \varepsilon(\mathrm{Y}) \leftrightarrow \delta(\mathrm{X}) \subseteq \mathrm{Y}$ then necessarily $\varepsilon$ and $\delta$ form an erosion/dilation pair.

We define $\varepsilon$ as trajectory-based erosion $\left(\ominus_{\tau}\right)$ and $\delta$ as trajectory-based dilation $\left(\oplus_{\tau}\right)$.

$$
\begin{array}{r}
\varepsilon=\ominus_{\tau}, \delta=\oplus_{\tau} \\
\text { Expression 2.31 }
\end{array}
$$

Proof: $X \subseteq \varepsilon(Y) \rightarrow \delta(X) \subseteq Y$

In descriptive terms, if a set $X$ of points belong to the set of points that is the result of trajectory-based erosion, an object $Y$ then the set of points $Y$ will necessarily be part of the result of the dilation of the set of points of $X$. We define $X$ as the set of points that is the result of eroding the object $Y$ by a structuring element $B$ :

$$
\begin{gathered}
\mathrm{X}=\mathrm{Y} \ominus_{\tau} \mathrm{B}=\bigcup_{\mathrm{k} \in[0 \ldots 1]}\left(\mathrm{Y} \ominus_{\tau(\mathrm{k})} \mathrm{B}\right) \\
=\left\{\mathrm{p} \in \mathrm{E}: \mathrm{p}=\tau^{-1}(\mathrm{q}, \mathrm{k}), \mathrm{q}=\underset{\mathbf{E x p r e s s i o n} \mathbf{2 . 3 2}}{=\operatorname{dist}_{\overrightarrow{\mathrm{C}}}(\mathrm{B}, \tau(\mathrm{Y}, \mathrm{k})) \cdot \overrightarrow{\mathrm{v}}} \wedge \mathrm{B}_{\mathrm{q}} \subseteq \tau(\mathrm{Y}, \mathrm{k})\right\}
\end{gathered}
$$

If $p$ is any point of the set $X, p$ is the minimal translation that the structuring element has to perform following the direction vector $\vec{v}$ for it to be placed on the inside of the object $Y$ touching at least one point $c$ of the object. The $c$ point of contact will depend on the parametric value $k$ used in the instantaneous basic operation. $Y$ is defined as the set of points that is the result of dilating the object $X$ by a structuring element $B$ :

$$
\begin{gathered}
\mathrm{Y}=\mathrm{X} \oplus_{\tau} \mathrm{B}=\bigcup_{\mathrm{k} \in[0 \ldots 1]}\left(\mathrm{X} \oplus_{\tau(\mathrm{k})} \mathrm{B}\right)= \\
=\left\{\mathrm{p} \in \mathrm{E}: \mathrm{p}=\tau^{-1}(\mathrm{q}, \mathrm{k}), \mathrm{q}=\operatorname{dist}_{\overrightarrow{\mathrm{w}}}(\mathrm{B}, \tau(\mathrm{X}, \mathrm{k})) \cdot \overrightarrow{\mathrm{w}} \wedge \mathrm{B}_{\mathrm{q}} \cap \tau(\mathrm{X}, \mathrm{k}) \neq \emptyset\right\}
\end{gathered}
$$


If $q$ is any point of the set $X, q$ is the minimum translation that the structuring element has to perform following the direction vector $\overleftarrow{W}$ for it to be placed on the outside of the object $X$ touching at least one point $d$ of the object.

If the direction vector $\vec{v}$ is equal to the direction vector $\overleftarrow{w}$ and the normalization of the trajectory-based erosion is homogeneous to that created in the dilation, point $p$ of the erosion will necessarily coincide with point $d$ of the dilation and contact point $c$ of the erosion will coincide with the computed point $q$ of the dilation. The dilated set $X$ s point will form part of the set of points that form the object $Y$ (Figure 5)

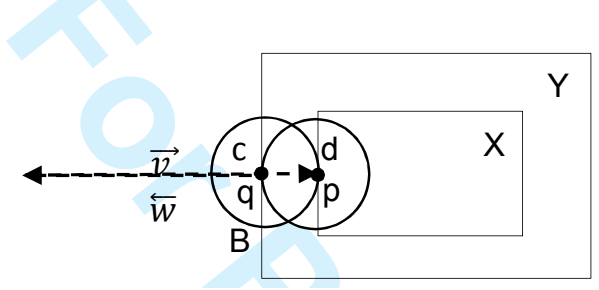

Figure 5. Proof of the Adjunction Theorem in trajectory-based operators

Thus, if the structuring elements' direction vectors are equal in the rotation and the trajectory-based dilation and the normalization of the parameterized spaces where the transformations have been carried out is homogeneous, every point generated by the erosion of a $Y$ object if it is dilated coincides with a point of the object $Y$, which demonstrates the involvement that $\mathrm{X} \subseteq \varepsilon(\mathrm{Y}) \rightarrow \delta(\mathrm{X}) \subseteq \mathrm{Y}$.

Proof: $\delta(X) \subseteq Y \rightarrow X \subseteq \varepsilon(Y)$

The dual involvement of the Adjunction Theorem means that involvement in both directions must be proven. Proof of this second involvement develops in the same terms as the first: If the direction vectors and the normalization of trajectory-based dilation is homogeneous to that created by erosion, the point calculated for trajectorybased dilation will necessarily coincide with the erosion's point of contact with the object and the dilation's point of contact with the object will coincide with the point calculated by erosion. Any point obtained by trajectory-based dilation of set $X$ (if eroded) coincides with an object $X$ point.

The general expression is proven by proving this second involvement, which states that the trajectory-based erosion and dilation operations are effectively morphological dilation and erosion. L.Q.Q.D operations.

\subsubsection{Morphological trajectory-based operators}

Morphological erosion and dilation form the basic composition of the so-called morphological filters that are obtained by combining the two basic operations. The following defines the specialization of the two most used filters although the entire extension of the morphological operation is covered by the definition of trajectorybased erosion and dilation. 
The classic morphological opening of $A$ by $B$ is obtained by eroding $A$ by $B$ and then dilating the resulting object by $B$. Formally:

$$
\begin{gathered}
A \circ \mathrm{B}=(\mathrm{A} \ominus \mathrm{B}) \oplus \mathrm{B} \\
\text { Expression 2.34 }
\end{gathered}
$$

In descriptive terms, the opening is the geometric locus of structuring element $B$ translations within the object $A$ :

$$
\mathrm{A} \circ \mathrm{B}=\bigcup_{\substack{\mathrm{B}_{\mathrm{x}} \subset \mathrm{A} \\ \text { Expresion }}} \mathrm{B}_{\mathrm{x}}
$$

In the opening there are two trajectory-based operations, an erosion and a dilation that generate different trajectories. The erosion places the structuring element on the inside of the object, touching its boundary while the dilation places it on the outside also touching its boundary (in this case the erosion's boundary). Morphological trajectory-based opening thus includes the definition of two functions of trajectory, $\tau_{1}$ and $\tau_{2}$ that will cover two sets of position-rotation values by describing the erosion and dilation trajectories that form the instantaneous morphological opening operation.

Trajectory-based opening will be given by applying the trajectory-based erosion operator followed by a trajectory-based dilation:

$$
\mathrm{A} \circ_{\tau} \mathrm{B}=\left(\mathrm{A} \ominus_{\tau_{1}} \mathrm{~B}\right) \bigoplus_{\tau_{2}}
$$

Trajectory-based closing

The closure of $A$ by $B$ is obtained by the dilation of $A$ by $B$, followed by the erosion of the resulting object by $B$. Formally:

$$
\underset{\text { Expression 2.37 }}{A \bullet B}
$$

The closure's geometrical interpretation is similar to the opening operator's. The difference is that the structuring element's movement is produced outside the object's boundary causing the contours to become smooth and the cracks or small holes to close. As happens with the opening, morphological trajectory-based closing includes the definition of two functions of trajectory $\tau_{1}$ and $\tau_{2}$, which define the dilation and erosion trajectories that form the closing operation.

The closing operator is defined as the application of a trajectory-based dilation followed by a trajectory-based erosion:

$$
\begin{gathered}
\mathrm{A} \cdot{ }_{\tau} \mathrm{B}=\left(\mathrm{A} \oplus_{\tau_{1}} \mathrm{~B}\right) \ominus_{\text {Expression } 2.38} \\
\tau_{\tau_{2}}
\end{gathered}
$$


Figure 6 shows the result of applying the complete trajectory-based closure of a rectangular object, carried out by a circular structuring element:

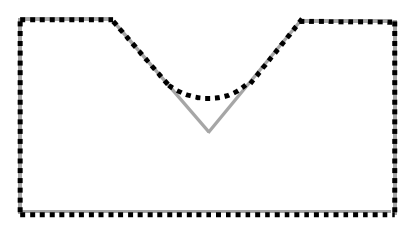

Figure 6. Trajectory-based closing of a rectangle with a circular object

\section{Discussion}

The DMM model provides a specialization of the classical morphological operations, allowing the generation of sequenced results and actions such as the movement of tools through trajectories. The definition of partial morphological operators allows the description of non-classical operations that are necessary from the point of view of design and manufacturing.

\subsection{DMM for manufacturing}

The action of removal of material in a machining process is related to the concept of trajectory-based morphological erosion. The machining can be interpreted as a morphological erosion operation in which a structural element transforms an object along a particular path. In an actual manufacturing process, the structuring element is the tool (T), the object is the piece to be transformed $(\mathrm{P})$, and the order defined in the trajectory function $\tau$ describes the machining path.

$$
\mathrm{P} \ominus_{\tau} \mathrm{H}=\bigcup_{\substack{\mathrm{k} \in[0 . .1] \\ \text { Expression } 3.1}}\left(\mathrm{P} \ominus_{\tau(\mathrm{k})} \mathrm{H}\right)
$$

This operation orderly obtains the tool centers when the tool contacts every point of the piece outline from the inside. The result of the morphological operation of trajectory-based erosion shows how the piece would end if the piece is manufactured using the defined tool and machining path.

Moreover, the machining trajectories may not just follow closed paths (equivalent to the trajectory-based morphological erosion) but also partial or open ones. The partial trajectory-based erosion is the operation that supports the possibility of partial machining.

Some first attempts to use mathematical morphology can be found in the literature. One example is the Topological modeling of the manufacturing process, who linked industrial machining with the concept of morphological erosion (Jimeno, 2003), (Jimeno et al., 2004).

\subsection{DMM for design}


The morphological trajectory-based erosion can be related with the actual manufacturing process, but also with the design process of objects, through the definition of virtual machining trajectories. This design philosophy allows the designer both to model the pieces and to define at a time the way in which they are made. So, the design and manufacturing processes are closely linked, which favors the quality in the general process of designing and manufacturing objects.

There are other models that closely relates both processes, such as the Trajectorybased design model, which bases object design on defining trajectories that are covered by modeling tools that simulate the material removed from the piece (Molina, 2002). Nevertheless, this model is not based on mathematical morphology.

Another basic design operation in a CAD/CAM system is the virtual 3D scanning, that is, the calculation of the virtual path followed by a touching feeler on a piece simulating the mechanical scanning process thereof. 3D scanning orderly obtains a sequence of the positions of the tool center. The calculation of the virtual scanning is closely related to one of the traditional problems in manufacturing systems: the offset calculation. The offset calculation is the process to determine the position to place a tool for machining a specific point in one piece, so that the tool does not collide with other already machined points. The equivalent morphological operation to virtual scanning is the morphological trajectory-based dilation. This operation obtains a compensated trajectory that describes the sequence of positions where the structuring element must be positioned to touch all the points in the object outline.

There has been several attempts to develop a mathematical morphology approach to calculate the offset of a shape. One example is (Molina-Carmona et al., 2007).

\subsection{Contributions}

One of the main contributions of the model is the integration of the processes of design and manufacture. The classic geometric models usually divide these processes in two phases: design phase and machining phase. On the one hand they provide tools and functionality for the design and, on the other hand, they define and plan the strategies for trajectory generation in other to machine the object.

The integrity and accuracy of the models depends on the tools used in the design and manufacturing phases but also on the procedures established for the connection of the two phases. In the best case, the independence of the processes can cause problems in accuracy and integrity of the manufactured parts.

The DMM tackles the problem of design and machining from a common perspective, linking both tasks in a single object definition process where design and trajectory generation and planning are indissoluble. To achieve continuity of this process, the design incorporates the definition of the machining paths, so that the trajectories are accurately described and the object is correctly machined. Moreover, the encapsulation of the design and machining processes allows the direct manufacturing of the object without generating additional paths, so it gives a great immediacy to the overall process.

The proposed model has another important value: the morphological operative is based on points sets, so the geometric data structures and the operations among points sets are intrinsically simple and efficient, compared to other much more complex models (such as CSG). 
The model is boundary-based, therefore no excessive computational resources are needed, because only the points in the boundary are processed. The use of boundarybased mathematical morphology is a classic way of decreasing the computational costy of the morphological operations. For instance, (Ragnemalm, 1992) and (Meijster et al., 2000) present techniques that apply morphological operations based on analytical calculations of distance between objects' boundary points. Van Vliet and Verwer present algorithms for the calculation of erosion, dilation, skeletonization and propagation of images based on the contour of figures (Van Vliet and Verwer, 1988) and (Wilkinson and Meijer, 1995) show a technique to classify microbiological organisms' images from applying morphological operations to the boundary pixels of organisms. All those examples belong to the field of image processing. Ours is an extension of these techniques to the field of CAD/CAM.

\section{Conclusions}

A geometric model has been presented that allows dynamic processes to be modeled from the formal framework that mathematical morphology presents. Therefore, a specialization of classic morphological operations has been defined, providing it with the determinism inherent in dynamic processes such as designing and manufacturing objects by machining. The specialization is based on a trajectory function, which uses translation and rotation transformations of objects to be transformed to facilitate the complete and orderly implementation of morphological operations.

Although the morphological framework is applied in object designing and manufacturing environments, its interest is not restricted to such processes. A clear example of an application beyond the object manufacturing process can be represented by image analysis. Since its very inception, mathematical morphology has been used in the analysis and filtering of images; this is not surprising as morphological filters are often used in numerous scientific disciplines. The model presented can contribute to these fields, regularizing morphological operations that give partial filtering and image ordering as results.

\section{References}

ISSM, 2011. International Symposium on Mathematical Morphology, 2011. Vancouver, BC, Canada.

Ghosh P.K. and Deguchi, K. (2008). Mathematics of shape description: A morphological approach to image processing and computer graphics, Wiley Editions, Singapore.

González R. and Woods, R. (2008). Digital Image Processing, Prentice Hall.

Jimeno, A.M. (2003). Modelado topológico del proceso de fabricación. Aplicación al compensado de herramienta. Ph.D. Dissertation. Universidad de Alicante. Available at: http://hdl.handle.net/10045/3653, accessed 12 february 2013.

Jimeno, A.M., Maciá, F. and García-Chamizo, J. (2004). “Trajectory-based morphological operators: a morphological model for tool path computation". In Proceedings of the international conference on algorithmic mathematics \& computer science, AMCS, Las Vegas. 
Meijster, A., Roerdink, J.B.T.M. and Hesselink, W.H. (2000). “A general algorithm for computing distance transforms in linear time". Mathematical Morphology and its Applications to Image and Signal Processing, Kluwer Acad. Publ., Dordrecht, 331340.

Molina, R. (2002). Modelo Superficie - Trayectoria. Un modelo geométrico para el diseño y la fabricación de objetos tridimensionales. Ph.D. Dissertation. Universidad de Alicante. Available at: http://hdl.handle.net/10045/3738, accessed 12 february 2013.

Molina-Carmona, R., Jimeno, A. and Rizo-Aldeguer, R. (2007). "Morphological offset computing for contour pocketing". Journal of manufacturing science and engineering, Vol. 129, No 2, pp. 400-406.

Plaza, A., Benediktsson, J.A., Boardman, J., Brazile, J., Bruzzone L. and Valls G. (2009). "Recent advances in techniques for hyperspectral image processing". Remote sensing of environment 113: 1. S110-S122.

Ragnemalm, I. (1992). "Fast erosion and dilation by contour processing and thresholding of distance maps, Pattern Recognition Letters 13, 161-166.

Salember, P., Wilkinson, M., Clairon, S. and Jean, P. (2009). "Connected operators. A review of region-based morphological image processing techniques”, IEEE Signal Processing Magazine 26, 136-157.

Sarabia, R., Jimeno, A. and Molina, R. (2010). "A Morphological Approach to the Design of Complex Objects”. Journal of Manufacturing Science and Engineering 132, 051003-7.

Serra, J. (1982). Image Analysis and Mathematical Morphology. Academic Press, London.

Serra, J. (1988). Image Analysis and Mathematical Morphology. Volume 2: Theoretical Advances. Academic Press, London.

Serra J. (2001). "Lecture Notes On Morphological Operators". First French-Nordic Summer Course in Mathematics. Uppsala University. Sweden.

Van Vliet, L. and Verwer, B. (1988). "A contour processing method for fast binary neighbourhood operations", Pattern Recognition Letters 727-36.

Wilkinson M. and Meijer, B. (1995). "DATAPLOT: A graphical display package for bacterial morphometry and fluorimetry data", Computer Methods and Programs in Biomedicine 47, 35-49. 